\title{
miR-193a-5p suppresses cell proliferation and induces cell apoptosis by regulating HOXA7 in human ovarian cancer
}

\author{
S. WANG ${ }^{1, *}$, Y. J. DIAO ${ }^{2}$, B. B. ZHU² \\ ${ }^{1}$ Department of Clinical Laboratory, The Fourth Hospital of Xian, Xian, China; ${ }^{2}$ Department of Clinical Laboratory, The First Affiliated Hospital \\ of Air Force Military Medical University, Xian, China \\ ${ }^{*}$ Correspondence: yanjingyaotkouw@163.com
}

Received July 30, 2019 / Accepted October 25, 2019

\begin{abstract}
Ovarian cancer is one of the most common malignancies in women in the world. MicroRNAs (miRNAs) were identified as a group of regulators that played important roles in the progression of cancer development. The main purpose of this study was to investigate the functional mechanism of microRNA-193a-5p (miR-193a-5p) in human ovarian cancer. Quantitative real-time polymerase chain reaction (qRT-PCR) was used to detect the RNA levels of miR-193a-5p and homeobox genes A7 (HOXA7). Western blot assay was performed to determine the protein level of HOXA7. The interaction between miR-193a-5p and HOXA7 was predicted by online software starBase v3.0, and then verified by the dual luciferase reporter assay. The cell proliferation and apoptosis rate were examined by 3-(4,5-dimethyl-2-thiazolyl)-2,5-diphenyl-2-H-tetrazolium bromide (MTT) and colony formation assay as well as flow cytometry analysis. We found out that the expression level of miR-193a-5p was decreased in human ovarian cancer tissues and cells. The overexpression of miR-193a-5p inhibited cell proliferation and induced apoptosis in human ovarian cancer. Interestingly, miR-193a-5p reduced the expression of HOXA7 by binding to 3'-untranslated region (3'-UTR) of HOXA7 mRNA. As expected, the knockdown of HOXA7 also suppressed cell proliferation and promoted apoptosis in human ovarian cancer. Besides, the upregulation of HOXA7 reversed the effect of miR-193a-5p on human ovarian cell proliferation and apoptosis. Our findings confirmed that miR-193a-5p inhibited cell proliferation and induced apoptosis through the downregulation of HOXA7 in human ovarian cancer, providing a theoretical value for the therapy of human ovarian cancer.
\end{abstract}

Key words: miR-193a-5p, HOXA7, proliferation, apoptosis, ovarian cancer

Ovarian cancer, the most lethal malignant gynecological cancer, led to an estimated 22,280 new cases and 14,240 deaths in 2016 in the US $[1,2]$. A lot of factors, including family history, reproductive history, the use of some drugs, smoking, and obesity, are closely related to ovarian cancer [3-6]. In our daily life, many ovarian cancer patients are not diagnosed before the advanced stage due to its asymptomatic nature at the early stage [7]. According to the statistics, only approximately $19 \%$ of patients are diagnosed at an early stage, which leads to a high mortality in ovarian cancer patients [8]. Surgery and platinum-based chemotherapy are main methods for the treatment of ovarian cancer, but there are still many defects about these two methods, such as toxicity, more inconvenience, and extra cost, and the controversy of these two methods is higher today than in the last 30 years [9]. Therefore, the research of new strategies is required to improve outcomes and hope for patients. In summary, it is imperative to study the molecular mechanism of ovarian cancer development.
MicroRNAs (miRNAs), with $\sim 22$ nucleotides, are non-coding RNAs that play pivotal roles at the transcriptional and post-transcriptional regulation by targeting 3'-untranslated region (3'-UTR) of mRNA [10, 11]. Due to directly targeting multiple mRNAs in cancers, miRNAs have been shown to regulate numerous cell progression, including proliferation, apoptosis, invasion, and autophagy [11-13]. miR-193a is an endogenous miRNA and reported to suppress cell growth in various human cancers, such as prostate cancer [14], osteosarcoma [15], and breast cancer [16]. miR-193a-5p, an isoform of miR-193a, was identified as a tumor suppressor in human cancers [17-19], but it is less studied compared with another miR-193a isoform miR-193a-3p. Moreover, miR-193a-5p and miR-193a-3p are rarely studied in human ovarian cancer.

Homeobox (HOX) genes encode conserved transcription factors that are involved in various cell progression, including proliferation, migration, and differentiation [20, 21]. In mammalians, HOX genes are divided into 4 subfami- 
lies, HOX A, B, C, D [22], and play vital roles in organogenesis [23]. In addition, HOX proteins play important roles in the development of human cancers, and the expression level of HOX is up- or downregulated in most solid tumors [24]. It is reported that HOXA7, a HOX gene, is related to the epithelial ovarian cancer cell differentiation and transport from the nucleus to cytoplasm during follicular maturation $[25,26]$. Recent evidence also showed that the knockdown of HOXA7 suppressed tumor growth and increased the chemosensitivity of tumors in human ovarian cancer [27]. These data indicated that HOXA7 is a positive regulator for ovarian cancer development, but its upstream components need to be investigated.

Here, we demonstrated that miR-193a-5p expression was decreased in human ovarian cancer cells. Moreover, the overexpression of miR-193a-5p inhibited cell proliferation and promoted apoptosis by downregulation of HOXA7 in human ovarian cancer.

\section{Patients and methods}

Tissues samples and cell culture. In total, 31 human ovarian cancer tissues and 31 matched adjacent normal tissues from the patients at the Department of Clinical Laboratory, the Fourth Hospital of Xi'an, Xincheng District, were collected. All tissues were immediately snap-frozen in liquid nitrogen and then stored at $-80^{\circ} \mathrm{C}$. This study was approved by the Ethics Committee of the Department of Clinical Laboratory, the Fourth Hospital of Xi'an.

A normal human ovarian surface epithelial cell line (HOSEpiC) was purchased from ScienCell Research Laboratories (San Diego, CA, USA) and human ovarian cancer cell lines (SKOV3 and OVCAR3) were purchased from American Tissue Culture Collection (ATCC, Manassas, VA, USA). A2780 cell line was obtained from the Affiliated Hospital of Guangdong Medical University (Zhanjiang, China). All cells were cultured in Dulbecco's Modified Eagle Medium (DMEM; Invitrogen, Carlsbad, CA, USA) supplemented with 10\% fetal bovine serum (FBS; Thermo Fisher Scientific, Waltham, MA, USA) and incubated in an incubator with $5 \% \mathrm{CO}_{2}$ at $37^{\circ} \mathrm{C}$.

Plasmid and transfection. miR-193a-5p mimic, miR-NC, small interfering RNA against HOXA7 (si-HOXA7\#1, si-HOXA7\#2, and si-HOXA7\#3), and si-NC were synthesized by GenePharma (Shanghai, China). For the overexpression of HOXA7, the full length HOXA7 was amplified by a pair of primers (forward primer, 5'-TCATTCCTCCTCGTCC-3' and reverse primer, 5'-ATGAGTTCTTCGTATTATGAACG-3') and inserted into pcDNA3.1 vector (GenePharma) using BamHI and EcoRI restriction enzymes to generate the overexpression vector. 3'-UTR sequence of HOXA7 and mutated binding sites of miR-193a-5p were cloned into pGL3 vector (Promega, Madison, WI, USA) for the dual luciferase reporter assay.

Cell transfection was performed by Lipofectamine 2000 transfection reagent (Invitrogen) according to the manufac- turer's protocol. The groups used in this study were listed as follows: miR-193a-5p, miR-NC, si-HOXA7, si-NC, miR-NC + HOXA7-Wt, miR-NC + HOXA7-Mut, miR-193a-5p + HOXA7-Wt, miR-193a-5p + HOXA7-Mut, pcDNA3.1NC, pcDNA3.1-HOXA7, miR-193a-5p + pcDNA3.1-NC, miR-193a-5p + pcDNA3.1-HOXA7.

RNA extraction and quantitative real-time polymerase chain reaction (qRT-PCR). Total RNA extraction was performed from human ovarian cancer tissues or cells using TRIzol (Invitrogen) according to the manufacturer's instructions. RNA concentration was determined using NanoDrop 2000 spectrophotometer (Thermo Fisher Scientific), followed by the reverse transcription reaction that was carried out using Prime Script RT reagent Kit (Takara, Shiga, Japan). qRT-PCR was carried out in a 96-well plate with SYBR green (Applied Biosystems, Foster City, CA, USA). U6 snRNA and glyceraldehyde 3-phosphate dehydrogenase (GAPDH) were used as endogenous genes for the relative expression of miR-193a-5p and HOXA7 analyzed by $2^{-\Delta \Delta \mathrm{Ct}}$ method. The reactions were initially denatured at $95^{\circ} \mathrm{C}$ for $5 \mathrm{~min}$, followed by 35 cycles of $95^{\circ} \mathrm{C}$ for $10 \mathrm{~s}$, and $60^{\circ} \mathrm{C}$ for $60 \mathrm{~s}$. The primes for miR-193a-5p, HOXA7, U6, and GAPDH were listed as follows: miR-193a-5p (forward primer, 5'-TATATGGGTCTTTGCGGGCG-3', reverse primer, 5'-GTGCAGGGTCCGAGGT-3'), HOXA7 (forward primer, 5'-TACCCCTGGATGCGGTCTT-3', reverse primer, 5'-CAGGTAGCGGTTGAAGTGGAA-3'), U6 (forward primer, 5'-TGCGGGTGCTCGCTTCGGCAGC-3', reverse primer, 5'-CCAGTGCAGGGTCCGAGGT-3'), GAPDH (forward primer, 5'CCGGGAAACTGTGGCGTGATGG-3', reverse primer, 5'AGGTGGAGGAGTGGGTGTCGCTGTT-3').

Western blot assay. After transfection for $48 \mathrm{~h}$, total proteins were isolated from cells using the cell lysis buffer (Takara Biotechnology Co., Ltd., Dalian, China). Protein concentration was determined by a NanoDrop 2000 spectrophotometer (Thermo Fisher Scientific). Equal amounts of proteins $(50 \mu \mathrm{g})$ were separated by dodecyl sulfate sodium salt polyacrylamide gel electrophoresis (SDS-PAGE, 10\%), and then transferred onto polyvinylidene difluoride (PVDF) membranes (Bio-Rad Laboratories, Inc., Hercules, CA, USA). The membranes were blocked by TBST (Tris-buffered saline containing $0.1 \%$ Tween-20) buffer containing 5\% non-fat milk at room temperature for $2 \mathrm{~h}$, followed by the incubation with primary antibodies $(1: 1000)$ at $4{ }^{\circ} \mathrm{C}$ overnight. After washing with TBST buffer for three times, the membranes were incubated with HRP-linked secondary antibodies (1:2000) at room temperature for $1 \mathrm{~h}$. After washing with TBST buffer for five times again, the proteins were visualized by Amersham ECL Plus ${ }^{\mathrm{TM}}$ Western Blotting Detection Reagents (Amersham Biosciences, Uppsala, Sweden) and analyzed with UVchem Detection Device (Biometra, Gottingen, Germany). Primary antibodies against HOXA7 (ab51235, Abcam, Cambridge, MA, USA), E-cadherin, (ab1416, Abcam), Snail (ab31787, Abcam), 
and GAPDH (ab9482, Abcam) were used in this study. The experiment was repeated for three times.

Cell proliferation, colony formation, and apoptosis assay. MTT assay was used to assess cell proliferation. In brief, after transfection, the cells were incubated for $24 \mathrm{~h}$, $48 \mathrm{~h}$, or $72 \mathrm{~h}$, respectively. Then, $20 \mu \mathrm{l}$ 3-(4,5-dimethyl-2thiazolyl)-2,5-diphenyl-2-H-tetrazolium bromide (MTT) solution ( $5 \mathrm{mg} / \mathrm{ml}$, Sigma, St. Louis, MO, USA) was added into each well, and then the cells were incubated for $4 \mathrm{~h}$ at $37^{\circ} \mathrm{C}$. After the supernatant was removed, $150 \mu \mathrm{l}$ dimethyl sulfoxide (DMSO, Sigma) was added to each well to dissolve the formazan crystal. Finally, the absorbance was determined at $490 \mathrm{~nm}$ using a microplate reader (Bio-Rad, Richmond, CA, USA).

Cell viability was measured by the colony formation assay. The cells transfected with miR-NC or miR-193a-5p were added into a plate and then cultured in the medium for 2 weeks. After the supernatant was removed, the cells were fixed with methanol. Next, the cells were washed with PBS and then stained with $0.1 \%$ crystal violet solution (Beyotime Biotechnology, Shanghai, China). Finally, a microscope was used to observe colonies.

Cell apoptosis assay was carried out using the Annexin V-Fluorescein (FITC) Apoptosis Detection Kit (Solarbio, Beijing, China). Firstly, the cells were collected and then washed with $1 \times$ Binding Buffer for 2 times. Subsequently, $1 \times$ Binding Buffer was used to resuspend the cells to a final concentration of $1 \times 10^{6}$ cells $/ \mathrm{ml}$. Next, $5 \mu \mathrm{l}$ of Annexin $\mathrm{V}$-FITC and $5 \mu \mathrm{l}$ of propidium iodide (PI) solution were added into $100 \mu \mathrm{l}$ of the cell suspension. Finally, the samples were detected by FACScan flow cytometry (BD Biosciences, San Jose, CA, USA) after incubation under the dark condition at room temperature.

The dual luciferase reporter assay. Bioinformatics analysis software starBase v3.0 was used to predict the target genes of miR-193a-5p. The 3'-UTR sequence of wild type HOXA7 (HOXA7-Wt) and its mutant (HOXA7-Mut) containing mutant binding sites of miR-193a-5p were cloned into reporter vector pGL3. Human ovarian cancer cells were co-transfected with miR-193a-5p and the reporter vector containing HOXA7-Mut or HOXA7-Wt. miR-NC was used as a negative control. After incubation for $48 \mathrm{~h}$, the luciferase activity was determined with Dual-Luciferase Reporter Assay System (Promega). The experiment was carried out three times.

Statistical analysis. Data were presented as the mean \pm S.E. of at least three independent experiments. A significant difference between the two groups was analyzed using Student's t-test. A p-value $<0.05$ was considered to be statistically significant. Each data group represents 3 biological replicates $\times 3$ technical replicates unless otherwise indicated.

\section{Results}

miR-193a-5p was downregulated in human ovarian cancer. To investigate the role of miR-193a-5p in human ovarian cancer, the qRT-PCR assay was performed to determine its expression. The results suggested that the expression level of miR-193a-5p was significantly decreased in human ovarian cancer tissues $(n=31)$ compared to matched adjacent normal tissues $(n=31$, Figure 1A). Similarly, obviously decreased expression of miR-193a-5p was observed in human ovarian cancer cells (SKOV3, OVCAR3, and A2780) compared with normal human cells (HOSEpiC, Figure 1B). These data indicated that miR-193a-5p might act as a negative regulator for ovarian cancer development.

The overexpression of miR-193a-5p suppressed proliferation and promoted apoptosis in human ovarian cancer cells. To further analyze the functional effect of miR-193a-5p on human ovarian cancer cells, SKOV3 and OVCAR3 cells were transfected with miR-NC or miR-193a-5p. Analysis of qRT-PCR confirmed that the expression level of
A

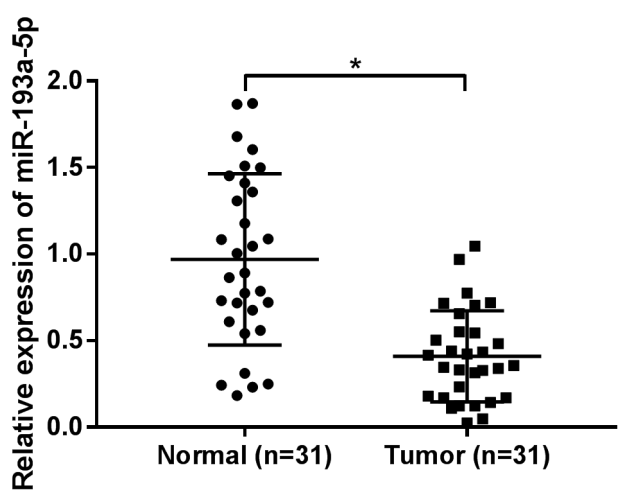

B

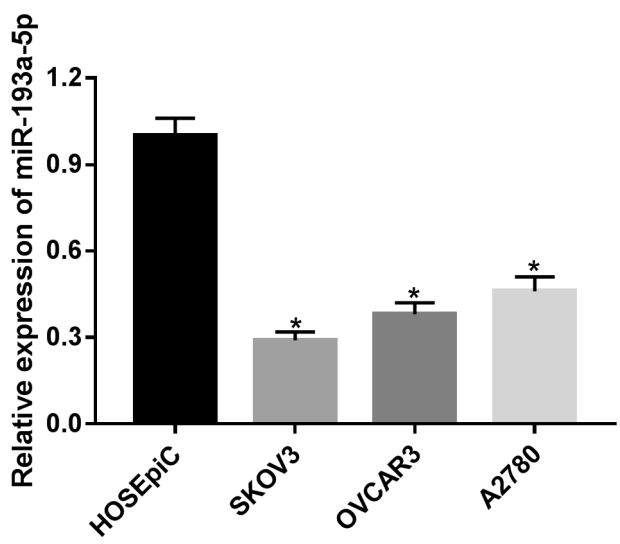

Figure 1. Expression of miR-193a-5p in human ovarian tissues and cells. A, B) qRT-PCR assay was performed to detect the RNA level of miR-193a-5p in human ovarian cancer tissues (A) and cells SKOV3, OVCAR3, and A2780 (B). Matched adjacent normal tissues and normal human cells (HOSEpiC) were used as a negative control, respectively. ${ }^{\star} \mathrm{p}<0.05$ 
miR-193a-5p was dramatically increased in the cells transfected with miR-193a-5p compared with the cells transfected with miR-NC (Figures 2A, 2B). Next, MTT assay and colony formation assay were used to examine cell proliferation. The results demonstrated that the overexpression of miR-193a-5p suppressed proliferation in both SKOV3 (Figures 2C, 2E) and OVCAR3 (Figures 2D, 2F). Meanwhile, the cell apoptosis rate was also detected by flow cytometry analysis. The data revealed that the upregulation of miR-193a-5p contributed to a significant increase of apoptosis rate in both SKOV3 (Figure 2G) and OVCAR3 (Figure 2H) cells. Therefore, the overexpression of miR-193a-5p inhibited cell proliferation and induced apoptosis in human ovarian cancer cells.

miR-193a-5p negatively regulated the expression of HOXA7 via targeting 3'-UTR of its mRNA. Bioinformatics software starBase v3.0 predicted that HOXA7 was a potential target gene of miR-193a-5p, so wild type HOXA7 (HOXA7-Wt) and mutant type HOXA7 (HOXA7-Mut) were cloned into luciferase reporter vector (Figure $3 \mathrm{~A}$ ). Then, the vectors containing HOXA7-Wt or HOXA7-Mut and miR-NC or miR-193a-5p were co-transfected into human ovarian cancer cells, and the dual luciferase reporter assay was performed. Analysis of the luciferase activity showed that the luciferase activity of HOXA7-Wt, but not HOXA7Mut, was strongly decreased by miR-193a-5p in both SKOV3 (Figure 3B) and OVCAR3 (Figure 3C) cells. Then, to investigate the effect of miR-193a-5p on HOXA7 expression, the expression level of HOXA7 was detected in cells transfected with miR-NC or miR-193a-5p. The results suggested that the transfection of miR-193a-5p significantly reduced the RNA and protein levels of HOXA7 in both SKOV3 (Figures 3D, 3F) and OVCAR3 (Figures 3E, 3G) cells. From these data, we concluded that miR-193a-5p decreased the expression of HOXA7 by targeting 3'-UTR of its mRNA.

Subsequently, the expression of HOXA7 was determined in human ovarian cancer tissues and normal tissues. As shown in Figure 3H, HOXA7 was significantly upregulated in human ovarian cancer tissues. Furthermore, we analyzed the relationship between miR-193a-5p and HOXA7. As expected, the HOXA7 level was negatively correlated with the miR-193a-5p level (Figure 3I). Besides, an increased HOXA7 level was observed in human ovarian cancer cells (Figure 3J). Therefore, HOXA7 might act as an oncogene in human ovarian cancer.
A

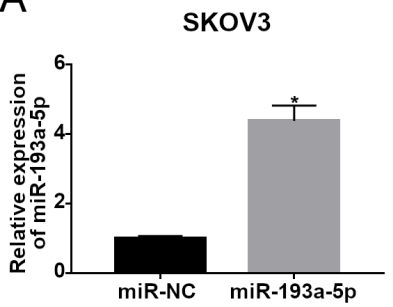

E
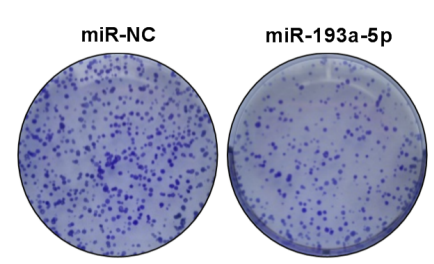

G

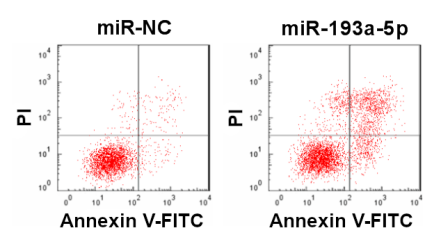

B

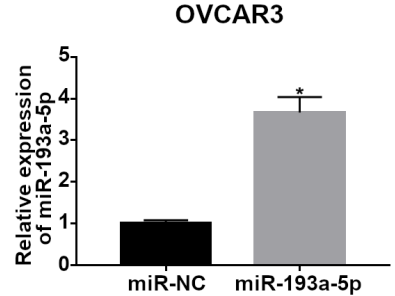

SKOV3

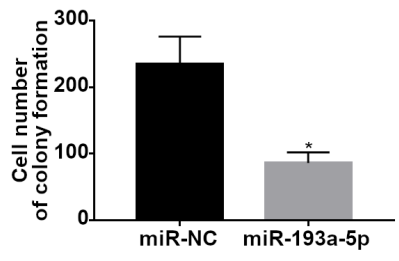

SKOV3

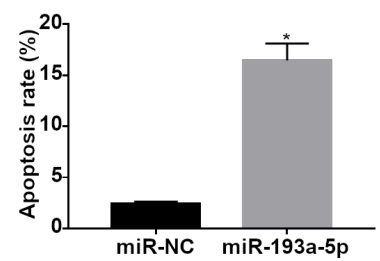

C

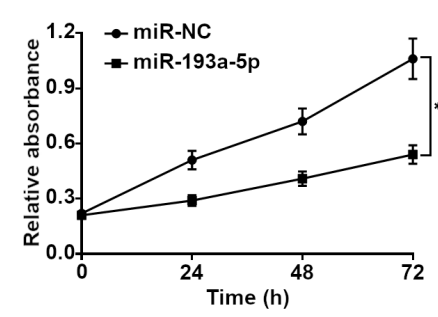

F
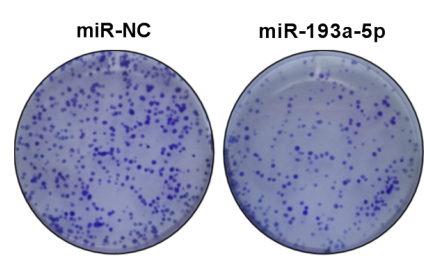

$\mathrm{H}$

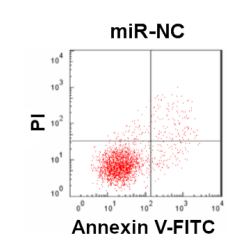

D

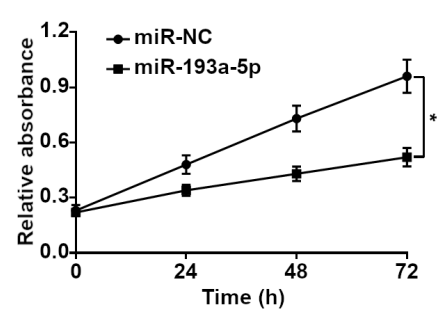

OVCAR3

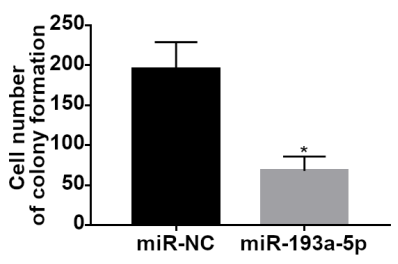

OVCAR3
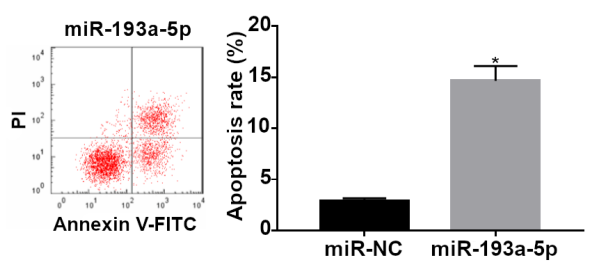

Figure 2. The overexpression of miR-193a-5p inhibited human ovarian cancer cell proliferation and induced apoptosis in vitro. A, B) The expression level of miR-193a-5p was detected in human ovarian cell SKOV3 and OVCAR3. C, D) MTT assay was performed to measure cell proliferation in human ovarian cell SKOV3 and OVCAR3. E, F) Colony formation assay was carried out to analyze the proliferation of SKOV3 and OVCAR3 cells. G, H) Cell apoptosis rate was assessed by flow cytometry analysis in SKOV 3 and OVCAR 3 cells. ${ }^{*} \mathrm{p}<0.05$ 
A

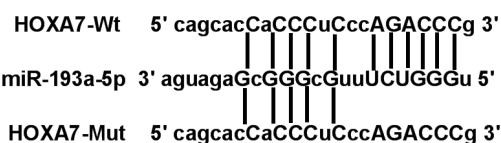

D

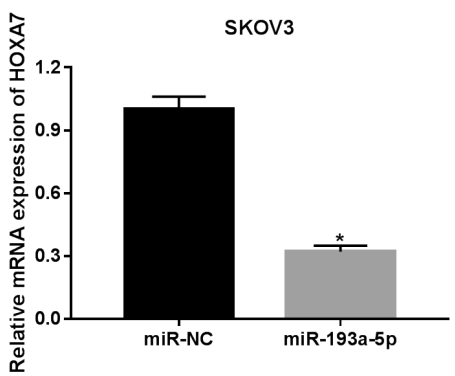

$\mathrm{F}$

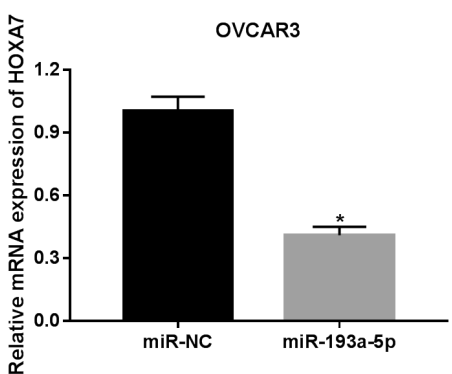

$\mathrm{H}$

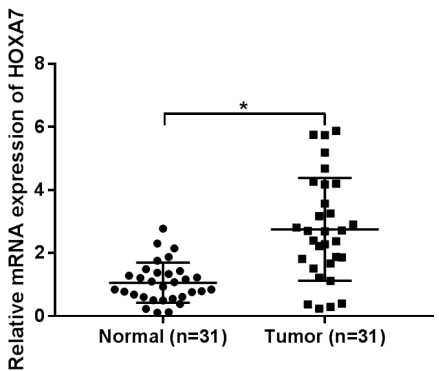

B

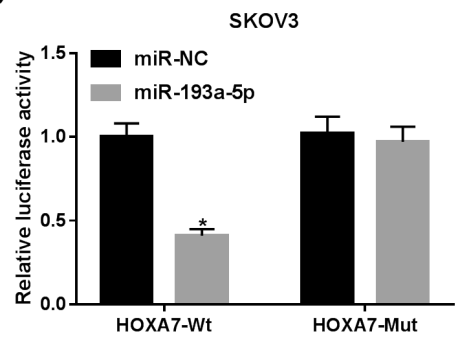

$E$

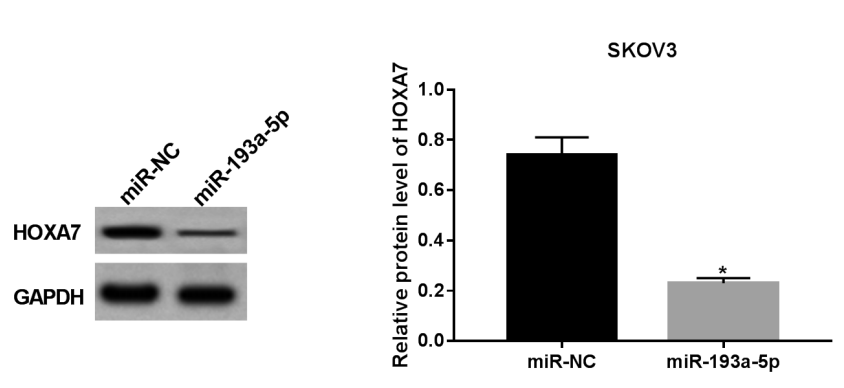

G
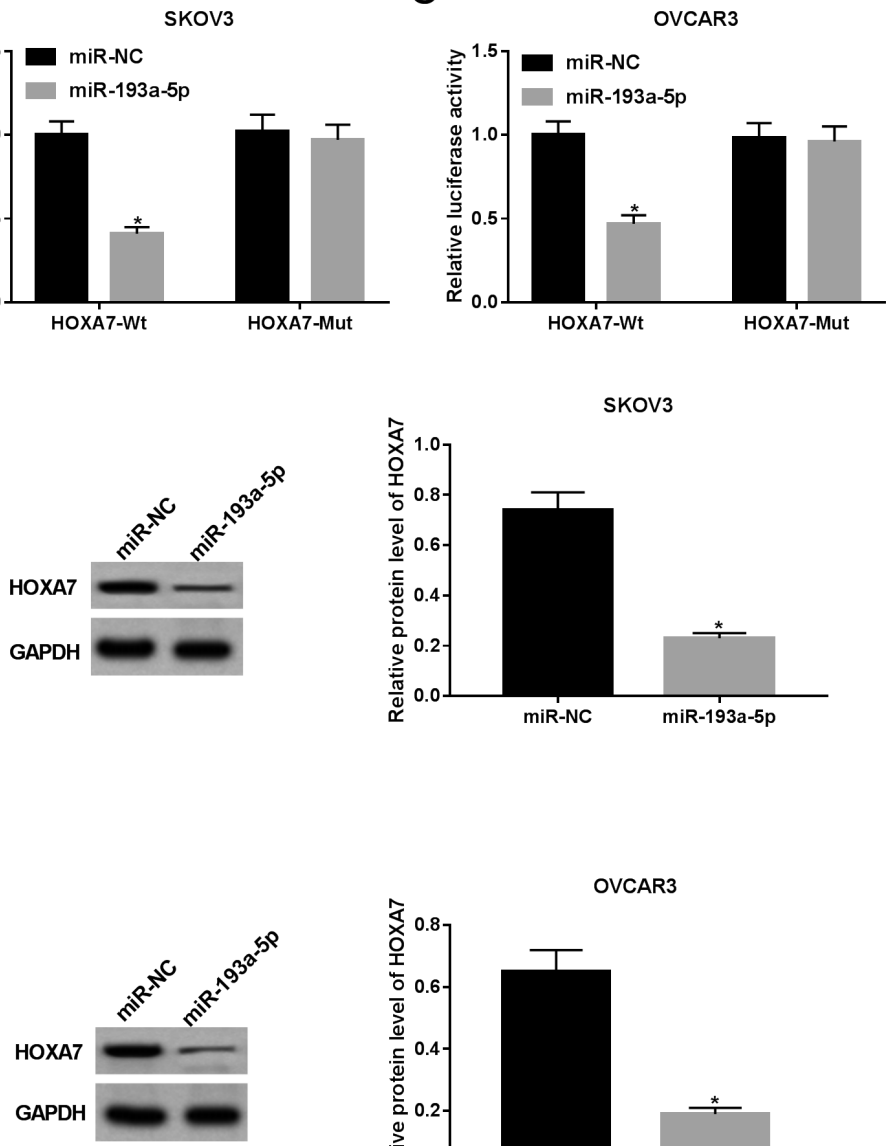

HOXA7-Wt

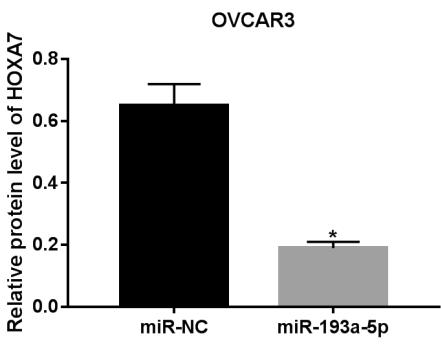

I

$J$
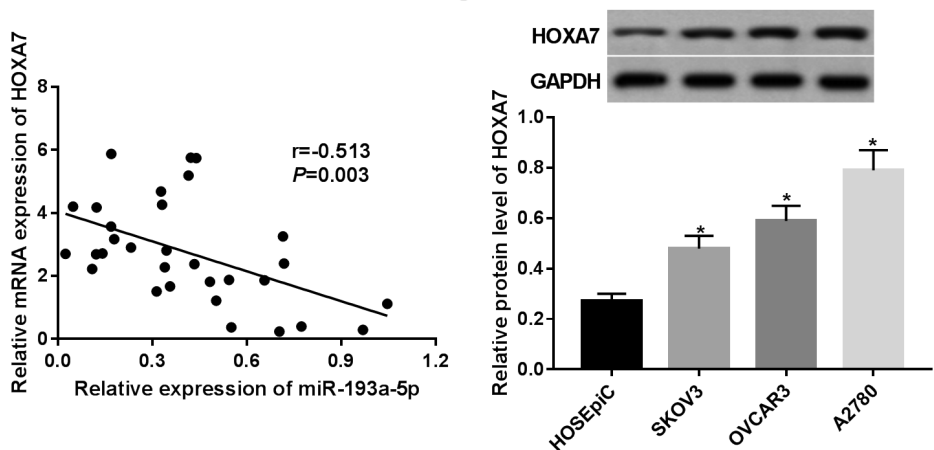

Figure 3. miR-193a-5p targeted and regulated HOXA7. A) The binding site of HOXA7 for miR-193a-5p was predicted by online software starBase v3.0. The mutated nucleotides were indicated in a horizontal line. $B, C$ ) The luciferase activity was determined in both SKOV3 and OVCAR3 cells cotransfected with miR-193a-5p and HOXA7-Wt or HOXA7-Mut, miR-NC was used as a negative control. D-G) The RNA and protein levels of HOXA7 were determined by qRT-PCR and western blot assay in SKOV3 and OVCAR3 cells transfected with miR-NC or miR-HOXA7, respectively. H) HOXA7 expression was detected in human ovarian tissues and matched normal tissues. I) The relationship between the HOXA7 level and the miR-193a-5p level was analyzed. J) HOXA7 expression was examined in human ovarian cells and normal cells. ${ }^{\star} \mathbf{p}<0.05$

The knockdown of HOXA7 inhibited proliferation and induced apoptosis in human ovarian cancer cells. To explore the effect of HOXA7 on human ovarian cancer, the cells were transfected with si-NC or si-HOXA7
(si-HOXA7\#1, si-HOXA7\#2, and si-HOXA7\#3) to deplete HOXA7. Western blot assay confirmed that the transfection of si-HOXA7 significantly downregulated the HOXA7 protein level in both SKOV3 (Figure 4A) and OVCAR3 
A

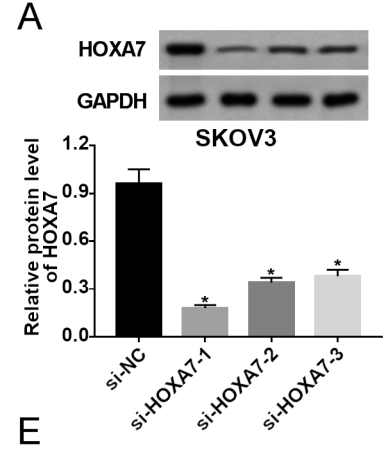

E

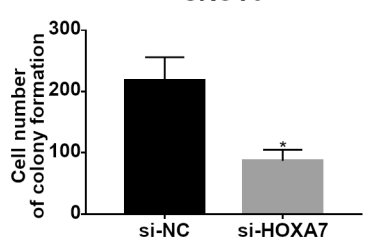

B
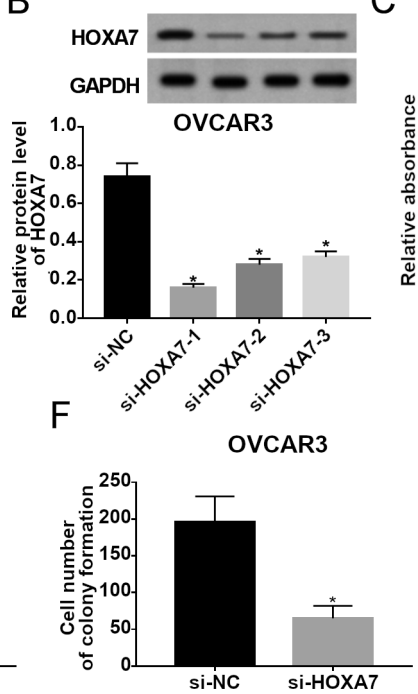

C

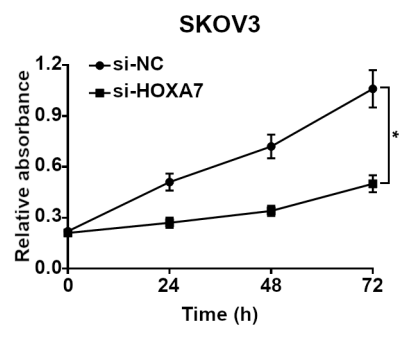

G

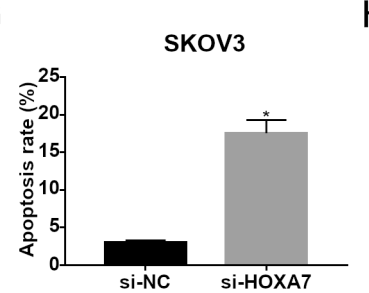

D

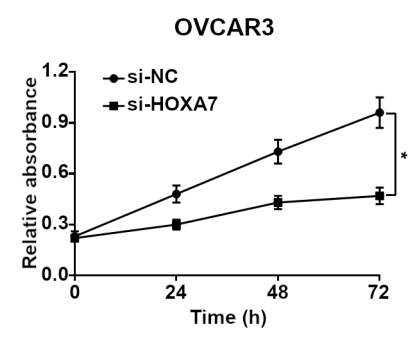

$\mathrm{H}$

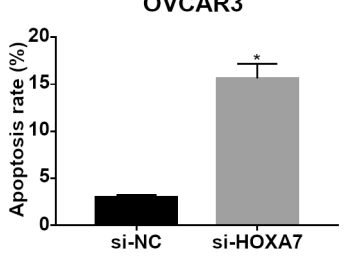

Figure 4. The downregulation of HOXA7 suppressed human ovarian cell proliferation and promoted apoptosis in vitro. A, B) The protein level of HOXA7 was detected by western blot in human ovarian cancer cell SKOV3 and OVCAR3. C, D) Cell proliferation was examined by MTT assay in SKOV3 and OVCAR3 cells. E, F) Colony formation assay was used to assess the proliferation in SKOV3 and OVCAR3 cells. G, H) Flow cytometry analysis was performed to measure cell apoptosis rate in SKOV3 and OVCAR3 cells. All cells were transfected with si-NC or si-HOXA7. ${ }^{\star}$ p $<0.05$

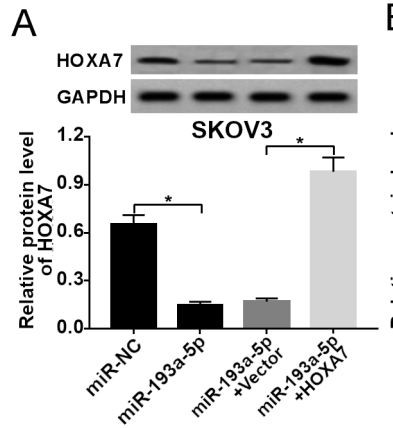

$\mathrm{E}$

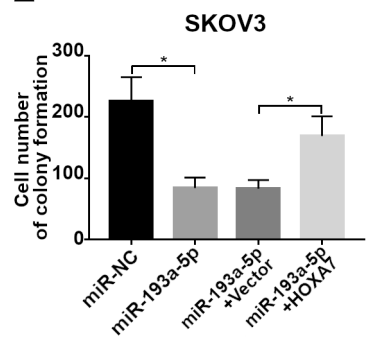

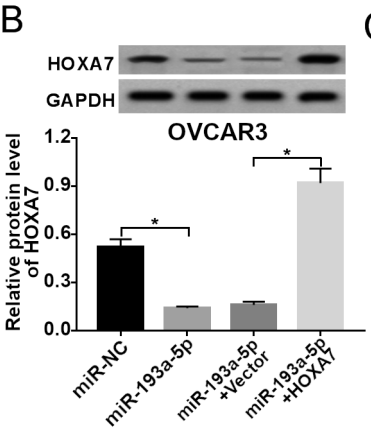

$\mathrm{F}$

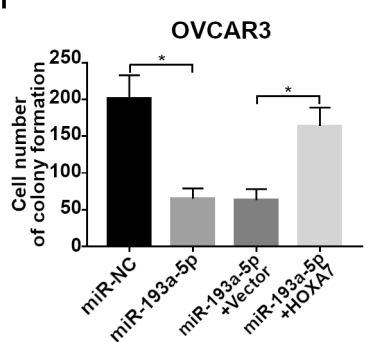

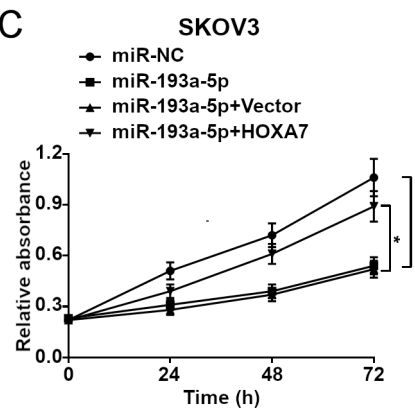

G

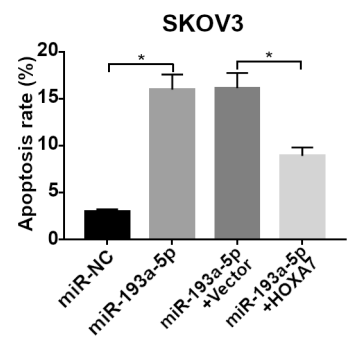

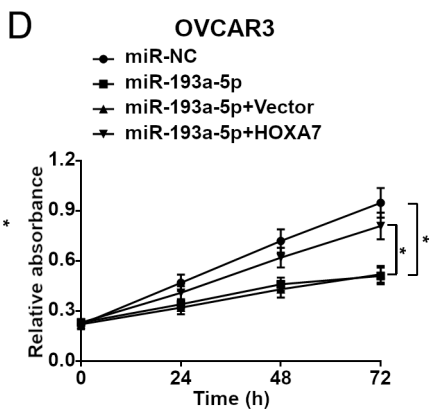

$\mathrm{H}$

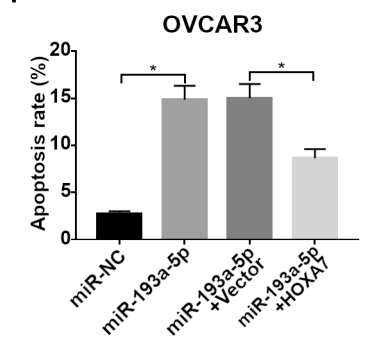

Figure 5. HOXA7 overexpression weakened the effect of miR-193a-5p on human ovarian cancer cells. A, B) SKOV3 and OVCAR3 cells were transfected with miR-NC, miR-193a-5p, miR-193a-5p + vector, miR-193a-5p + HOXA7. The protein level of HOXA7 was detected by western blot. C, D) MTT assay was performed to assess the cell proliferation in both SKOV3 and OVCAR3 cells. E, F) Colony formation assay was used to detect cell proliferation in both SKOV 3 and OVCAR 3 cells. G, H) Cell apoptosis rate was measured by flow cytometry analysis in both SKOV 3 and $\mathrm{OVCAR} 3$ cells. ${ }^{\star}$ p $<0.05$

(Figure 4B) cells. Next, MTT assay and colony formation assay were performed to assess the cell proliferation in cells transfected with si-NC or si-HOXA7. The results indicated that the knockdown of HOXA7 suppressed proliferation in both SKOV3 (Figures 4C, 4E) and OVCAR3 (Figures 4D, 4F) cells. Besides, we also measured cell apoptosis rate and found that the downregulation of HOXA7 obviously promoted apoptosis in both SKOV3 (Figure 4G) and OVCAR3 (Figure $4 \mathrm{H}$ ) cells. On the other hand, we analyzed the effects of HOXA7 on the levels of E-cadherin and Snail. As shown in Supplementary Figure S1 A-C, HOXA7 knockdown reduced the E-cadherin level and elevated the Snail level in 
both SKOV3 and OVCAR3 cells. These results indicated that the depletion of HOXA7 suppressed cell proliferation and promoted apoptosis in human ovarian cancer.

The upregulation of HOXA7 reversed the effect of miR-193a-5p on human ovarian cancer cells. Based on the above results, it was speculated that HOXA7 played a vital role in the miR-193a-5p pathway. To verify this possibility, the cells were transfected with miR-193a-5p or miR-193a-5p + HOXA7. Western blot assay confirmed that enforced expression of miR-193a-5p dramatically decreased the HOXA7 protein level, whereas the transfection of HOXA7 eliminated this change in both SKOV3 (Figure 5A) and OVCAR3 (Figure 5B) cells. Then, cell viability was assessed to analyze the effect of HOXA7 on miR-193a-5p-mediated cell progression. The data suggested that the upregulation of miR-193a-5p significantly inhibited cell proliferation and induced apoptosis, whereas overexpression of HOXA7 abrogated these effects in both SKOV3 (Figures 5C, 5E, 5G) and OVCAR3 (Figures 5D, 5F, 5H). Thus, the overexpression of HOXA7 reversed the effect of miR-193a-5p on human ovarian cancer cell proliferation and apoptosis.

\section{Discussion}

Ovarian cancer is one of the most threatening gynecologic cancers in women. miRNAs play pivotal roles in many aspects of human cancers, including ovarian cancer. In this study, we indicated that the upregulation of miR-193a-5p inhibited cell proliferation and induced apoptosis through downregulating HOXA7. Our results revealed the underlying mechanism and theoretical basis for the therapy of ovarian cancer.

Although previous evidence showed that the expression of miR-193a-5p was decreased in many human cancers $[18,28,29]$, the role of miR-193a-5p in ovarian cancer was almost unexplored. Until 2018, a paper mentioned that miR-193a-5p expression was downregulated in epithelium ovarian cancer (EOC) patients compared with benign ovarian tumor patients [30]. Consistent with this data, our results also suggested that the expression level of miR-193a-5p was decreased in human ovarian cancer tissues and cells. Thus, miR-193a-5p may play a negative role in the progression of ovarian cancer development.

The effect of miR-193a-5p on human ovarian cancer has not been reported yet, but present evidence confirmed that miR-193a-5p inhibited cell growth in various human cancers, including human osteosarcoma [18], colon cancer [19], and non-small-cell lung cancer [31]. In this study, we confirmed that the upregulation of miR-193a-5p suppressed ovarian cancer cell proliferation and promoted apoptosis. These functional effects were similar to that in other cancers. From these data, it was concluded that miR-193a-5p negatively regulated the growth of ovarian cancer.

In human cancers, miRNAs affect cell growth by targeting and regulating downstream mRNA [9]. Thus, we explored target genes of miR-193a-5p using online tool starBase v3.0 and found an interesting gene HOXA7. Next, the dual luciferase reporter assay was used to verify this interaction. Consistent with the prediction, the result demonstrated that miR-193a-5p bound to 3'-UTR of HOXA7 mRNA. Previous evidence showed that miRNAs decreased the expression level of the gene by targeting it $[32,33]$. Then, we detected the mRNA and protein levels of HOXA7 in human ovarian cancer cells after the overexpression of miR-193a-5p. The results suggested that the upregulation of miR-193a-5p significantly decreased the expression level of HOXA7, this result was accordant with known knowledge. These data revealed that miR-193a-5p downregulated HOXA7 expression via targeting 3'-UTR of HOXA7 mRNA.

HOXA7 is a transcription factor and related to various human cancers. For example, it induced cell proliferation via regulating estrogen receptor-alpha and epidermal growth factor receptor in breast cancer and human granulosa cells, respectively $[34,35]$. In liver cancer, the upregulation of HOXA7 promoted cell proliferation, migration, invasion, and tumor growth [36]. Besides, HOXA7 also induced cell proliferation and reduced the chemosensitivity of cells in human ovarian cancer [27]. These data suggested that HOXA7 positively regulated the progression of some cancers including ovarian cancer. In this study, we depleted HOXA7 and found that ovarian cancer cells exhibited the phenotypes of decreased proliferation and increased apoptosis rate. These results were consistent with previous data. Furthermore, the previous data demonstrated that the ration of Snail/E-cadherin affected cell growth and drug resistance in ovarian cancer cells, and HOXA7 positively regulated the level of E-cadherin [37, 38]. In this study, we analyzed the levels of E-cadherin and Snail and after HOXA7 knockdown. As expected, E-cadherin expression was downregulated and Snail expression was upregulated by HOXA7 knockdown. Therefore, we concluded that HOXA7 positively regulated cell growth in human ovarian cancer.

Based on the above results, it was speculated that miR-193a-5p regulated cell growth by affecting HOXA7 in human ovarian cancer. Then, we enforced the expression of HOXA7 under the condition of miR-193a-5p overexpression and found that the upregulation of HOXA7 reversed the effect of miR-193a-5p on ovarian cancer cell progression, including proliferation and apoptosis. Taken together, miR-193a-5p regulated cell growth by downregulation of HOXA7 in human ovarian cancer.

In summary, we data established an association between miR-193a-5p and HOXA7 and suggested that miR-193a-5p suppressed cell proliferation and promoted apoptosis via targeting HOXA7 in human ovarian cancer. These findings are helpful for the understanding of the molecular mechanism of ovarian cancer development and provide theoretical value for the treatment of ovarian cancer.

Supplementary information is available in the online version of the paper. 


\section{References}

[1] BANERJEE S, TIAN T, WEI Z, SHIH N, FELDMAN MD et al. The ovarian cancer oncobiome. Oncotarget 2017; 8: 36225-36245. https://doi.org/10.18632/oncotarget.16717

[2] TORRE LA, BRAY F, SIEGEL RL, FERLAY J, LORTETTIEULENT J et al. Global cancer statistics, 2012. CA Cancer J Clin 2015; 65: 87-108. https://doi.org/10.3322/caac.21262

[3] SCHOUTEN LJ, RIVERA C, HUNTER DJ, SPIEGELMAN D, ADAMI HO et al. Height, body mass index, and ovarian cancer: a pooled analysis of 12 cohort studies. Cancer Epidemiol Biomarkers Prev 2008; 17: 902-912. https://doi. org/10.1158/1055-9965.EPI-07-2524

[4] SUEBLINVONG T, CARNEY ME. Current understanding of risk factors for ovarian cancer. Curr Treat Options Oncol 2009; 10: 67-81. https://doi.org/10.1007/s11864-009-0108-2

[5] TWOROGER SS, FAIRFIELD KM, COLDITZ GA, ROSNER BA, HANKINSON SE. Association of oral contraceptive use, other contraceptive methods, and infertility with ovarian cancer risk. Am J Epidemiol 2007; 166: 894-901. https://doi.org/10.1093/aje/kwm157

[6] WHITTEMORE AS, BALISE RR, PHAROAH PD, DICIOCCIO RA, OAKLEY-GIRVAN I et al. Oral contraceptive use and ovarian cancer risk among carriers of BRCA1 or BRCA2 mutations. Br J Cancer 2004; 91: 1911-1915. https://doi. org/10.1038/sj.bjc.6602239

[7] FORBES C, SHIRRAN L, BAGNALL AM, DUFFY S, TER RIET G. A rapid and systematic review of the clinical effectiveness and cost-effectiveness of topotecan for ovarian cancer. Health Technol Assess 2001; 5: 1-110. https://doi. org/10.3310/hta5280

[8] Eskander RN, Bristow RE. Epithelial ovarian cancer, low malignant potential, and sex cord stromal tumors of the ovary. Springer New York 2015 https://doi.org/10.1007/978-14939-1976-5_1

[9] Gourley C, Walker JL, Mackay HJ. Update on Intraperitoneal Chemotherapy for the Treatment of Epithelial Ovarian Cancer. American Society of Clinical Oncology Educational Book 36 (October 29, 2018) 143-151. https://doi. org/10.1200/EDBK_158927

[10] LAGES E, IPAS H, GUTTIN A, NESR H, BERGER F et al. MicroRNAs: molecular features and role in cancer. Front Biosci (Landmark Ed) 2012; 17: 2508-2540. https://doi. org/10.2741/4068

[11] LIU B, LI J, CAIRNS MJ. Identifying miRNAs, targets and functions. Brief Bioinform 2014; 15: 1-19. https://doi. org/10.1093/bib/bbs075

[12] AREDIA F, SCOVASSI AI. A new function for miRNAs as regulators of autophagy. Future Med Chem 2017; 9: 25-36. https://doi.org/10.4155/fmc-2016-0173

[13] VENTURA A, JACKS T. MicroRNAs and cancer: short RNAs go a long way. Cell 2009; 136: 586-591. https://doi. org/10.1016/j.cell.2009.02.005

[14] LING Z, WANG X, TAO T, ZHANG L, GUAN H et al. Involvement of aberrantly activated HOTAIR/EZH2/miR-193a feedback loop in progression of prostate cancer. J Exp Clin Cancer Res 2017; 36: 159. https://doi.org/10.1186/s13046017-0629-7
[15] WANG SN, ZHAO XQ, YU B, WANG BW. miR-193a inhibits osteogenic differentiation of bone marrow-derived stroma cell via targeting HMGB1. Biochem Biophys Res Commun 2018; 503: 536-43. https://doi.org/10.1016/j. bbrc.2018.05.132

[16] XIE F, HOSANY S, ZHONG S, JIANG Y, ZHANG F et al. MicroRNA-193a inhibits breast cancer proliferation and metastasis by downregulating WT1. PLoS One 2017; 12: e0185565. https://doi.org/10.1371/journal.pone.0185565

[17] JIN L, LI H, WANG J, LIN D, YIN K et al. MicroRNA-193a$5 p$ exerts a tumor suppressor role in glioblastoma via modulating NOVA1. J Cell Biochem 2019; 120: 6188-97. https:// doi.org/10.1002/jcb.27906

[18] PU Y, ZHAO F, CAI W, MENG X, LI Y et al. MiR-193a-3p and miR-193a-5p suppress the metastasis of human osteosarcoma cells by down-regulating Rab27B and SRR, respectively. Clin Exp Metastasis 2016; 33: 359-72. https://doi. org/10.1007/s10585-016-9783-0

[19] SHIRAFKAN N, SHOMALI N, KAZEMI T, SHANEHBANDI D, GHASABI $M$ et al. microRNA-193a-5p inhibits migration of human HT-29 colon cancer cells via suppression of metastasis pathway. J Cell Biochem 2018 https://doi. org/10.1002/jcb. 28164

[20] GORSKI DH, WALSH K. The role of homeobox genes in vascular remodeling and angiogenesis. Circ Res 2000; 87: 865-872. https://doi.org/10.1161/01.res.87.10.865

[21] MCGINNIS W, KRUMLAUF R. Homeobox genes and axial patterning. Cell 1992; 68: 283-302. https://doi. org/10.1016/0092-8674(92)90471-n

[22] KRUMLAUF R. Hox genes in vertebrate development. Cell 1994; 78: 191-201. https://doi.org/10.1016/00928674(94)90290-9

[23] VITIELLO D, KODAMAN PH, TAYLOR HS. HOX genes in implantation. Semin Reprod Med 2007; 25: 431-436. https:// doi.org/10.1055/s-2007-991040

[24] BHATLEKAR S, FIELDS JZ, BOMAN BM. HOX genes and their role in the development of human cancers. J Mol Med (Berl) 2014; 92: 811-823. https://doi.org/10.1007/s00109014-1181-y

[25] NAORA H, MONTZ FJ, CHAI CY, RODEN RB. Aberrant expression of homeobox gene HOXA7 is associated with müllerian-like differentiation of epithelial ovarian tumors and the generation of a specific autologous antibody response. Proc Natl Acad Sci U S A 2001; 98: 15209-15214. https://doi.org/10.1073/pnas.011503998

[26] OTA T, CHOI KB, GILKS CB, LEUNG PC, AUERSPERG $\mathrm{N}$. Cell type- and stage-specific changes in HOXA7 protein expression in human ovarian folliculogenesis: possible role of GDF-9. Differentiation 2006; 74: 1-10. https://doi. org/10.1111/j.1432-0436.2006.00053.x

[27] LIU S, LEI H, LUO F, LI Y, XIE L. The effect of lncRNA HOTAIR on chemoresistance of ovarian cancer through regulation of HOXA7. Biol Chem 2018; 399: 485-497. https://doi. org/10.1515/hsz-2017-0274

[28] CHOU NH, LO YH, WANG KC, KANG CH, TSAI CY et al. MiR-193a-5p and -3p Play a Distinct Role in Gastric Cancer: miR-193a-3p Suppresses Gastric Cancer Cell Growth by Targeting ETS1 and CCND1. Anticancer Res 2018; 38: 3309-3318. https://doi.org/10.21873/anticanres.12596 
[29] ZHANG P, JI DB, HAN HB, SHI YF, DU CZ et al. Downregulation of miR-193a-5p correlates with lymph node metastasis and poor prognosis in colorectal cancer. World J Gastroenterol 2014; 20: 12241-12248. https://doi.org/10.3748/wjg. v20.i34.12241

[30] REN X, ZHANG H, CONG H, WANG X, NI H et al. Diagnostic Model of Serum miR-193a-5p, HE4 and CA125 Improves the Diagnostic Efficacy of Epithelium Ovarian Cancer. Pathol Oncol Res 2018; 24: 739-744. https://doi. org/10.1007/s12253-018-0392-X

[31] YU T, LI J, YAN M, LIU L, LIN H et al. MicroRNA-193a$3 p$ and $-5 p$ suppress the metastasis of human non-small-cell lung cancer by downregulating the ERBB4/PIK3R3/mTOR/ S6K2 signaling pathway. Oncogene 2015; 34: 413-423. https://doi.org/10.1038/onc.2013.574

[32] BARTEL DP. MicroRNAs: genomics, biogenesis, mechanism, and function. Cell 2004; 116: 281-297. https://doi. org/10.1016/s0092-8674(04)00045-5

[33] HE L, HANNON GJ. MicroRNAs: small RNAs with a big role in gene regulation. Nat Rev Genet 2004; 5: 522-531. https://doi.org/10.1038/nrg1379
[34] ZHANG Y, CHENG JC, HUANG HF, LEUNG PC. Homeobox A7 stimulates breast cancer cell proliferation by up-regulating estrogen receptor-alpha. Biochem Biophys Res Commun 2013; 440: 652-627. https://doi.org/10.1016/j. bbrc.2013.09.121

[35] ZHANG Y, HUANG Q, CHENG JC, NISHI Y, YANASE T et al. Homeobox A7 increases cell proliferation by up-regulation of epidermal growth factor receptor expression in human granulosa cells. Reprod Biol Endocrinol 2010; 8: 61. https://doi.org/10.1186/1477-7827-8-61

[36] TANG B, QI G, SUN X, TANG F, YUAN S et al. HOXA7 plays a critical role in metastasis of liver cancer associated with activation of Snail. Mol Cancer 2016; 15: 57. https://doi. org/10.1186/s12943-016-0540-4

[37] LEE YJ, WU CC, LI JW, OU CC, HSU SC et al. A rational approach for cancer stem-like cell isolation and characterization using CD44 and prominin-1(CD133) as selection markers. Oncotarget 2016; 7: 78499-78515. https://doi. org/10.18632/oncotarget.12100

[38] OTA T, GILKS CB, LONGACRE T, LEUNG PC, AUERSPERG N. HOXA7 in epithelial ovarian cancer: interrelationships between differentiation and clinical features. Reprod Sci. 2007; 14(6): 605-14. https://doi. org/10.1177/1933719107307781 


\section{miR-193a-5p suppresses cell proliferation and induces cell apoptosis by regulating HOXA7 in human ovarian cancer}

S. WANG ${ }^{1, *}$, Y. J. DIAO ${ }^{2}$, B. B. $\mathrm{ZHU}^{2}$

Supplementary Information

A

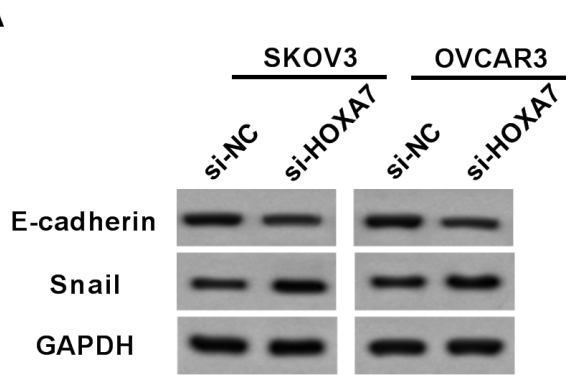

B

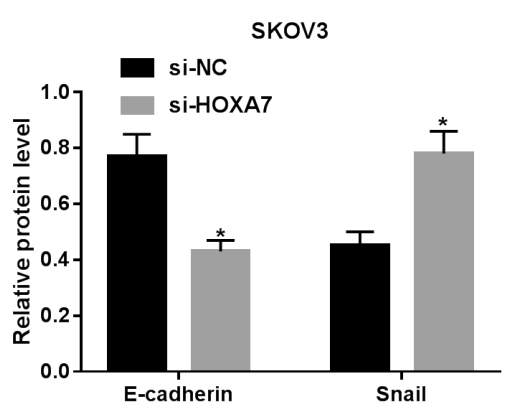

C

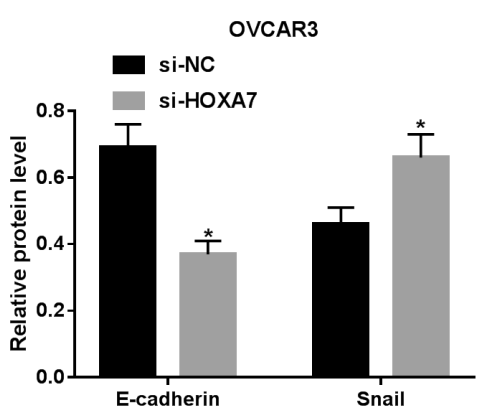

Supplementary Figure S1. HOXA7 knockdown downregulated the levels of E-cadherin and Snail. A-C) Western blot assay was performed to detect the levels of E-cadherin and Snail in SKOV3 and OVCAR3 cells transfected with si-NC or si-HOXA7. 